\title{
3D FEM temperature distribution analysis of the human eye exposed to laser radiation
}

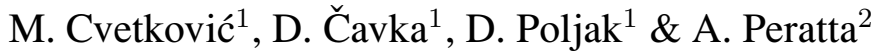 \\ ${ }^{1}$ University of Split, Faculty of Electrical Engineering, \\ Mechanical Engineering and Naval Architecture, Croatia \\ ${ }^{2}$ Wessex Institute of Technology, $U K$
}

\begin{abstract}
This paper is an extension of a previously published work on thermal modelling of the human eye exposed to radiation by various lasers. The 2D model is now extended to $3 \mathrm{D}$ in order to give a more realistic representation of the human eye model when irradiated by laser. The model is based on space-time dependent Pennes' bio-heat transfer equation solved by the finite elements method featuring Galerking-Bubnov procedure. The results for the temperature field inside the eye could prove to be useful in minimising the possible damage to intraocular tissues during the ophthalmological procedures and also later in analysis of laser-tissue interaction mechanisms. Also, analysis of various parameters on the calculated temperature distribution is carried out.
\end{abstract}

\section{Introduction}

The last few decades have witnessed an expansion of laser usage in various ophthalmological operations. Nowadays, medical lasers cover wavelengths from ultraviolet to the infrared parts of the electromagnetic spectrum.

An increasing number of laser uses and spread of different types of eye surgeries has led to a need for quantitatively understanding basic laser-tissue interaction. Due to this fact, a number of models [1-5] were developed to predict the various processes inside the eye, with the goal of helping ophthalmologists in minimizing the possible damage to ocular tissue while operating with laser.

The interaction of light with tissue results in either a transmission, reflection, scattering, or absorption of this light. In modelling laser-tissue interaction, the most important among these is absorption, since absorbed light is re-emitted as radiant 
energy or transformed into heat which results in an increase of the temperature field inside tissue.

Blood flow is a key element in thermoregulation of the living organism, however, in the human eye, it cannot regulate the heating inside the ocular tissue. Due to this fact, a thermal model of the human eye is required.

In order to derive a model which describes laser thermal effects, heat transfer needs to be taken into account. In biological tissue, this is achieved by using the Pennes' bioheat transfer equation, which takes into account different types of transfer processes.

This model is an extension to a previous $2 \mathrm{D}$ model [6] and aims to give more a realistic representation of the temperature field inside the human eye when subjected to a radiation of $694.3 \mathrm{~nm}$ Ruby laser, widely used in ophthalmology operations, such as posterior capsulotomy (after-cataract), peripheral iridotomy (treatment of glaucoma), for retinal photocoagulation $[7,8]$.

\section{Model description}

\subsection{The human eye}

The eye is a very complex optical system. It measures about $24 \mathrm{~mm}$ in length and $23 \mathrm{~mm}$ in diameter. We have assumed the eye to be a solid structure of given dimensions, consisting of eight homogeneous tissues, namely, cornea, aqueous humour, cilliary body, lens, vitreous humour, retina, choroid and sclera.

The extent and degree of tissue damage depends on laser parameters such as wavelength, power density, exposure time, spot size, and repetition rate, but also on optical tissue properties like absorption and scattering coefficients, and thermal tissue properties such as heat capacity and thermal conductivity.

Figure 1 shows a flow chart with the parameters required when modelling lasereye thermal interaction.

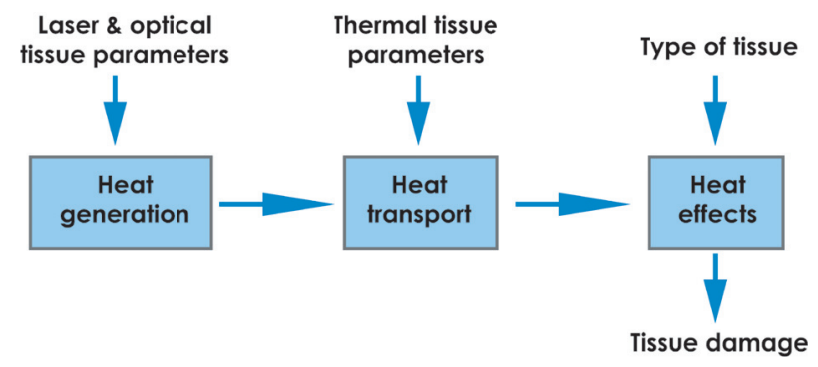

Figure 1: Parameters required for modelling thermal interaction.

Heat generation inside tissue is determined by laser parameters and optical tissue properties while the transfer of heat to the surrounding tissues is characterized 
Table 1: Thermal and optical properties of various human eye tissues.

\begin{tabular}{r|llllll}
\hline Tissue type & $\begin{array}{l}\text { Volumetric } \\
\text { perfusion } \\
W_{b}\end{array}$ & $\begin{array}{l}\text { Internal } \\
\text { umetric } \\
\text { generation } \\
Q_{m}\end{array}$ & $\begin{array}{l}\text { vol- } \\
\text { heat }\end{array}$ & $\begin{array}{l}\text { Thermal } \\
\text { conductivity } k\end{array}$ & $\begin{array}{l}\text { Specific heat } \\
\text { capacity } C\end{array}$ & $\begin{array}{l}\text { Abs.coef. } \\
(694.3 \mathrm{~nm})\end{array}$ \\
\hline Vitreous humour & 0 & 0 & 0.594 & 3997 & 2 \\
Lens & 0 & 0 & 0.400 & 3000 & 9.5 \\
Aqueous humour & 0 & 0 & 0.578 & 3997 & 8.4 \\
Cornea & 0 & 0 & 0.580 & 4178 & 124 \\
Sclera & 0 & 0 & 0.580 & 4178 & 358.55 \\
Cilliary body & 2700 & 690 & 0.498 & 3340 & 8.167 \\
Choroid & 0 & 0 & 0.530 & 3840 & 16847 \\
Retina & 35000 & 10000 & 0.565 & 3680 & 44000 \\
\hline
\end{tabular}

by the thermal tissue properties. These parameters are given in Table 1, taken primarily from [9].

In biological tissues, absorption is mainly due to the presence of water molecules, proteins, pigments, and other macromolecules, and is governed by Lambert-Beer's law. As the absorption coefficient strongly depends on the wavelength of laser radiation as seen from Fig. 2, different tissues will strongly absorb only at certain wavelengths. This is the principle by which the medical lasers work.

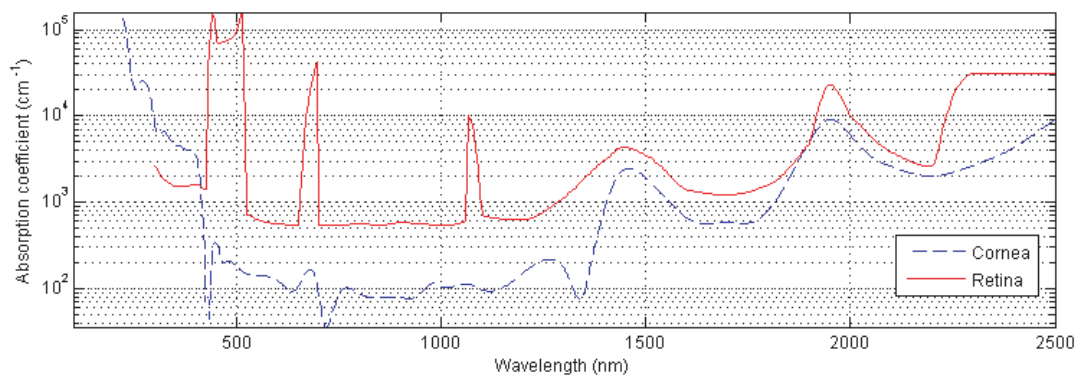

Figure 2: Absorption coefficient for cornea and retina.

Strong absorption in the infrared region of the spectrum is primarily attributed to water molecules, while proteins and pigments are main absorbers in the UV and visible range of the spectrum [10-12].

Values for absorption coefficient of various eye tissues are given in Table 1, taken from [13]. 


\subsection{Heat transfer}

The mathematical model of the human eye is based on the Pennes' bioheat transfer equation [14]. This equation is the foundation for all mathematical analysis in the field of bioheat transfer [15]. In Pennes' model, the rate of tissue temperature increase is given by the sum of the net heat conduction into the tissue, metabolic heat generation, and the heating (cooling) effects due to arterial blood flow:

$$
\rho C \frac{\partial T}{\partial t}=\nabla(k \nabla T)+W_{b} C_{p b}\left(T_{a}-T\right)+Q_{m}+H
$$

Bioheat equation is extended with the new term $H$ representing heat generated inside the tissue due to laser radiation.

Bioheat equation is supplemented with natural boundary condition equations for cornea and sclera, respectively:

$$
\begin{gathered}
-k \frac{\partial T}{\partial n}=h_{c}\left(T-T_{a m b}\right)+\sigma \epsilon\left(T^{4}-T_{a m b}^{4}\right) \in \Gamma_{1} \\
-k \frac{\partial T}{\partial n}=h_{s}\left(T-T_{a}\right) \in \Gamma_{2}
\end{gathered}
$$

where $k$ is specific tissue thermal conductivity, $h_{c}$ heat transfer coefficient of cornea, $h_{s}$ heat transfer coefficient of sclera, $\sigma$ Stefan-Boltzmann constant, $\epsilon$ emissivity of the corneal surface, $T_{a m b}$ temperature of the ambient air anterior to the cornea, and $T_{a}$ arterial blood temperature taken to be $36.7^{\circ} \mathrm{C}$. The value for $h_{c}$ is taken to be $14 \mathrm{~W} / \mathrm{m}^{2 \circ} \mathrm{C}, h_{s}$ is $65 \mathrm{~W} / \mathrm{m}^{2 \circ} \mathrm{C}$, emissivity of the cornea is 0.975 , and the value of ambient temperature is $25^{\circ} \mathrm{C}$.

Eqs. (2) and (3) describe the thermal exchange between cornea and surrounding air due to convection and radiation, and thermal exchange between sclera and ocular globe due to convection only, respectively. Second term on the right handside of Eq. (2) is approximated by $\sigma \epsilon T_{F}^{3}\left(T-T_{a m b}\right)$, where $T_{F}$ is the melting temperature of the substance [2]. The value for $T_{F}$ is taken to be $100^{\circ} \mathrm{C}$.

\subsection{Heat generation - laser source modeling}

Laser energy $H(r, z, t)$, absorbed by the eye tissue at the $n^{t h}$ node with cylindrical coordinates $(r, z)$, is given by a product

$$
H(r, z, t)=\alpha I(r, z, t)
$$

where $\alpha$ is the wavelength dependent absorption coefficient of the specific tissue, and $I$ is the power density or intensity of the $n^{t h}$ node, given by

$$
I(r, z, t)=I_{0} \exp \left(-\frac{2 r^{2}}{w^{2}}-\alpha z\right) \exp \left(-\frac{8 t^{2}}{\tau^{2}}\right)
$$

where $I_{0}$ is the incident value of intensity, $w$ is the beam waist, and $\tau$ is the pulse duration. 
For a given laser parameters, the irradiance at the cornea is calculated from $I_{c}=4 P / d_{c}^{2} \pi$, where $P$ is laser power and $d_{c}$ is a beam diameter on the cornea. Taking into account the focusing action of the lens, diameter of the image and irradiance on the retina is calculated from

$$
d_{r}=2.44 \frac{\lambda f}{d_{p}} \text { and } I_{r}=I_{c} \frac{d_{p}^{2}}{d_{r}^{2}}
$$

where $\lambda$ is laser wavelength, $f$ focal distance of the lens, and $d_{p}$ diameter of pupillary opening. From these values, we now interpolate the intermediate values for the beam width and irradiance along the beam path.

The parameters for Ruby laser, used in our calculations were; pulse duration 10 $\mathrm{ms}$, laser power $0.15 \mathrm{~W}$, laser beam diameter on cornea $8 \mathrm{~mm}$, and pupil diameter $7.3 \mathrm{~mm}$. Laser pulse was sampled in five $2 \mathrm{~ms}$ steps, and in each of these steps, calculation of temperature field distribution has been done.

\subsection{Finite element method}

Analytical solution of the bioheat equation is limited to a few simple geometries with high degree of symmetry, but using the FEM, problems on complex geometries such as human eye could be solved.

The equation (1) is discretized in three spatial dimensions and solved using the weak formulation and the Galerkin-Bubnov procedure. A total number of 148,664 tetrahedral elements and 27,624 nodes were generated using the GID mesh generator and solving part was done by algorithm written in MATLAB.

The bioheat equation is first solved for the steady-state case, i.e. when no external sources are present, and latter, these results are used as initial conditions in the time domain analysis with included laser source.

\section{Results}

\subsection{Steady-state case}

The steady-state temperature distribution in the human eye is shown in Fig. 3. The bottom part of the same figure shows a comparison of temperature along the pupillary axis between the previous 2D model and this one.

Steady-state results are in good agreement with number of papers [2, 4, 16-19].

\section{2 $694.3 \mathrm{~nm}$ Ruby laser analysis}

A temperature of $87.3^{\circ} \mathrm{C}$ is obtained on a node on vitreo-retinal boundary, and a maximum temperature of $106.5^{\circ} \mathrm{C}$ is obtained on a lens node. The previous model noted maximum temperatures on scleral nodes, while a more realistic situation would be to have maximum temperatures on the retina, due to a high value for the absorption coefficient of the retina for this wavelength. For this reason, $694.3 \mathrm{~nm}$ 


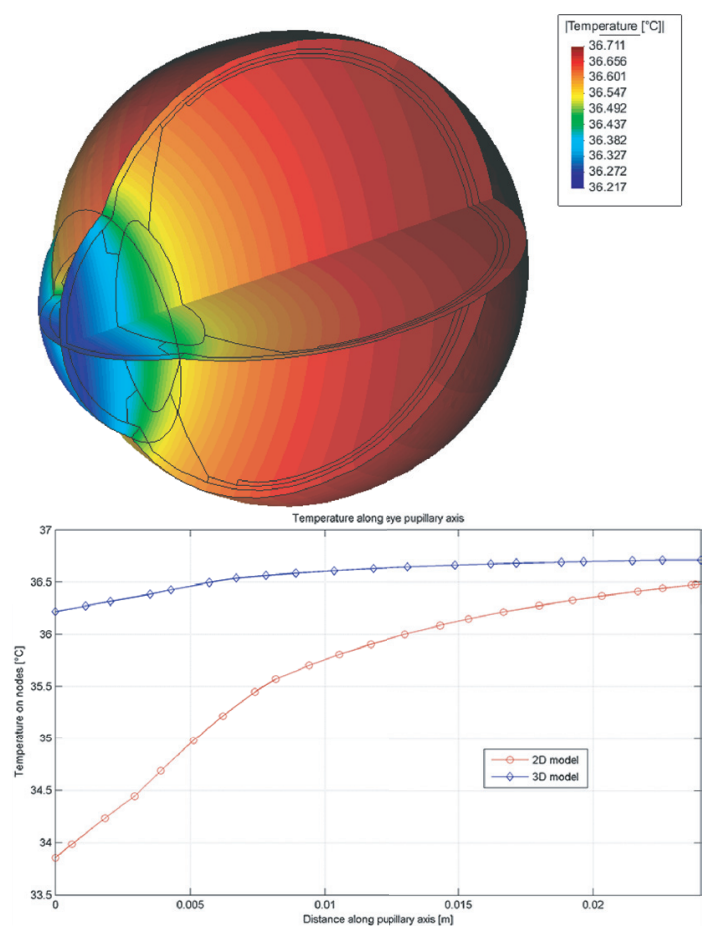

Figure 3: Steady-state temperature field in the human eye and temperature along the eye pupillary axis (current and previous model).

Ruby laser is used for retinal photocoagulation. The reason for this discrepancy probably lies in the fact that the previous model used isoparametric elements for the boundary (sclera and cornea), therefore not exactly taking into account the heat flux from these boundary elements. Also, pulse duration in this model is set to $\tau=0.01 \mathrm{~s}$.

Fig. 4 shows the temperature field distribution in the eye on a horizontal and vertical slice along pupillary axis, and a maximum temperature on the vitreousretina boundary can be seen in Fig. 5, detailing the temperature field around the posterior part of the eye.

Fig. 6 shows five equidistant transversal plane slices of the temperature field in the eye and a temperature distribution on the anterior part of the lens tissue.

\subsection{Parameter analysis}

The following is the analysis of various parameters on the calculated temperature distribution. Tables 2-4 depict the effect of the beam diameter on the cornea, pupillary opening and ambient temperature, on temperature rise on nodes number 3, 290, 1925, 26589, 26925 and 27245, situated on the corneo- 


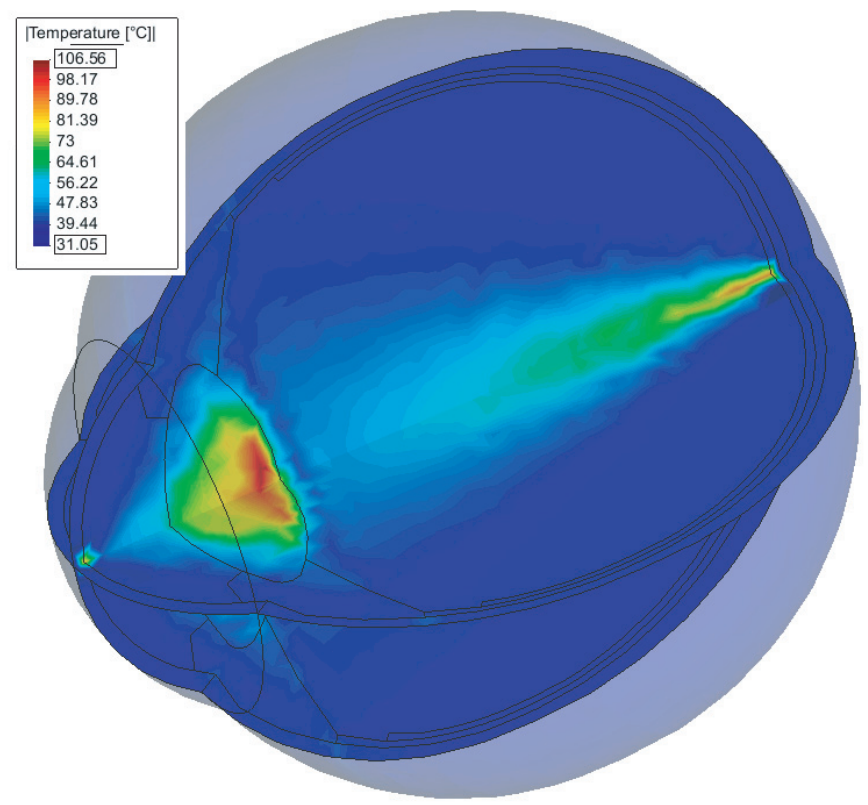

Figure 4: Temperature field distribution in the eye. Horizontal and vertical slice along pupillary axis.

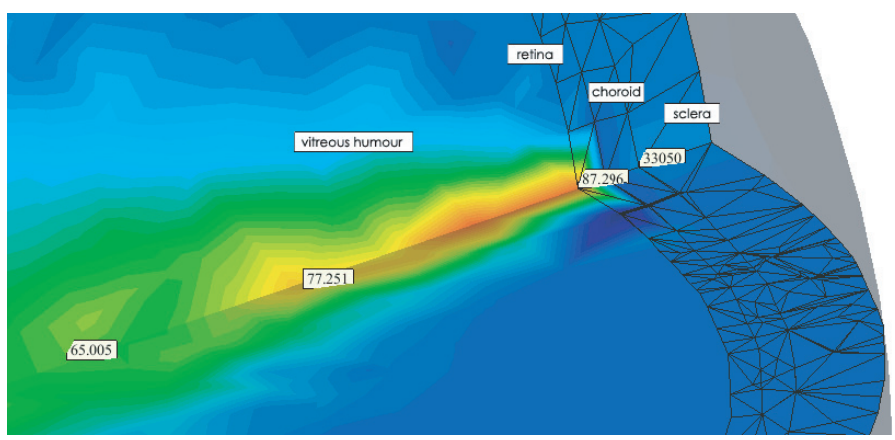

Figure 5: Detail of the temperature field on the posterior part of the eye.

aqueous, aqueous-lens, lens-vitreous, vitreo-retinal, retina-choroid, and choroidsclera boundary, respectively.

One can note that the laser beam diameter on the cornea has a significant effect on the temperature obtained, as previously reported by [2]. The reason for this is the fact that the same amount of laser energy concentrated on a smaller spot surface, results in a more absorbed power, and hence higher temperatures. 


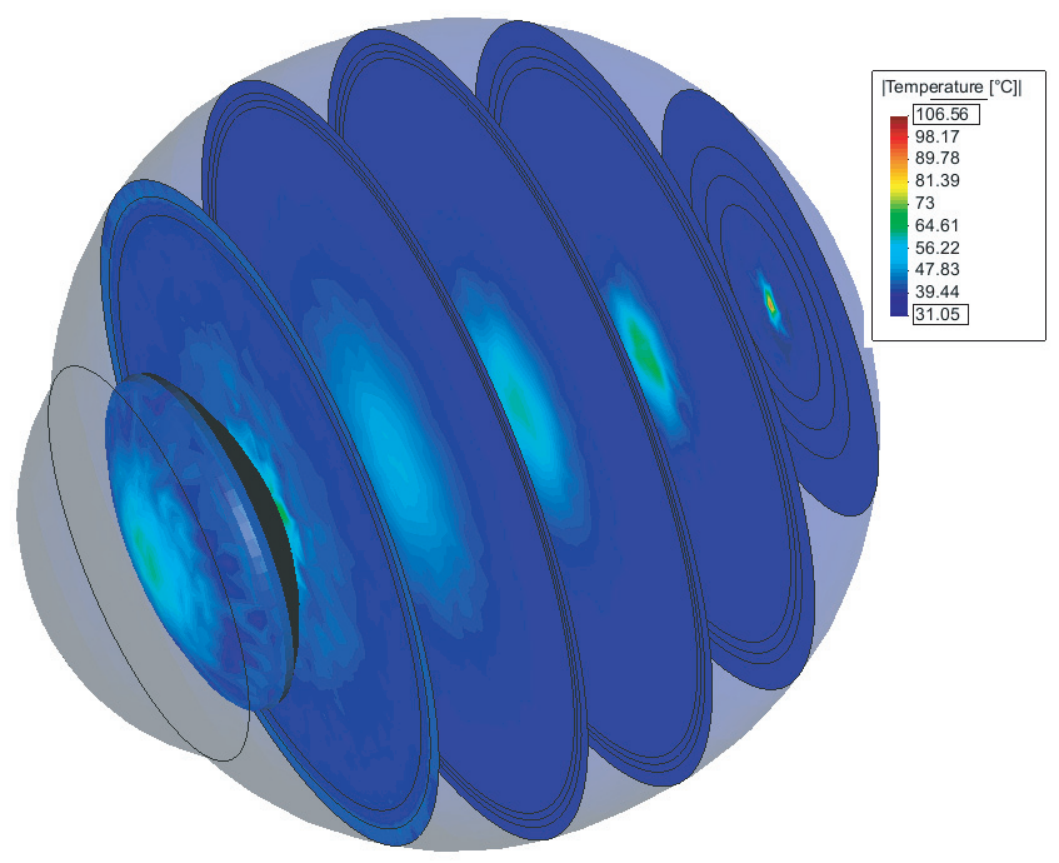

Figure 6: Transversal plane slices of the temperature field in the eye and temperature distribution on the anterior part of the lens tissue.

Analysis on the pupillary opening showed that a wider pupil results in a higher temperatures. This was expected since the pupillary opening controls the total amount of light entering the eye.

Finally, Table 4 shows that the ambient temperature has some effect on the anterior parts of the eye, while almost none has a direct effect on the posterior parts. This is the same as according to [16].

Table 2: The effect of the beam diameter on the cornea on the temperature distribution on the selected nodes in the eye.

\begin{tabular}{r|ccccccc}
\hline$d_{c}(\mathrm{~mm})$ & Node 3 & Node 290 & Node 1925 & Node 26589 & Node 26925 & Node 27245 & Max. Temp. \\
\hline 8 & 84.78 & 62.28 & 74.94 & 87.3 & 33.9 & 38.58 & 106.56 \\
6 & 122.5 & 82.44 & 104.8 & 126.6 & 31.71 & 40.03 & 161.04 \\
4 & 230.4 & 140 & 190.2 & 239.1 & 25.46 & 44.18 & 316.68 \\
\hline
\end{tabular}


Table 3: The effect of the pupillary opening on the temperature distribution on the selected nodes in the eye.

\begin{tabular}{r|ccccccc}
\hline$d_{p}(\mathrm{~mm})$ & Node 3 & Node 290 & Node 1925 & Node 26589 & Node 26925 & Node 27245 & Max. Temp. \\
\hline 8 & 105.5 & 73.47 & 91.81 & 106.9 & 32.78 & 39.39 & 137.36 \\
7.3 & 84.78 & 62.28 & 74.94 & 87.3 & 33.9 & 38.58 & 106.56 \\
4 & 40.71 & 38.91 & 40.1 & 42.05 & 36.43 & 36.88 & 42.966 \\
\hline
\end{tabular}

Table 4: The effect of the ambient temperature on the temperature distribution on the selected nodes in the eye.

\begin{tabular}{r|ccccccc}
\hline $\begin{array}{r}T_{a m b} \\
\left({ }^{\circ} \mathrm{C}\right)\end{array}$ & Node 3 & Node 290 & Node 1925 & Node 26589 & Node 26925 & Node 27245 & Max. Temp. \\
\hline 20 & 84.54 & 62.1 & 74.83 & 87.25 & 33.86 & 38.54 & 106.44 \\
25 & 84.78 & 62.28 & 74.94 & 87.3 & 33.9 & 38.58 & 106.56 \\
30 & 85.02 & 62.46 & 75.05 & 87.34 & 33.94 & 38.62 & 106.68 \\
\hline
\end{tabular}

\section{Conclusion}

Temperature distribution analysis of the human eye exposed to radiation by a 694.3 $\mathrm{nm}$ Ruby laser has been made. A 3D model was developed in order to give a more realistic representation of the human eye. Pennes' bioheat equation extended with the term representing the heat generated inside the tissue due to laser radiation is solved using the Finite Element Method. Results gave a temperature of $87.3^{\circ} \mathrm{C}$ on the retina, while a maximum temperature was achieved in the lens tissue, different from the previous model. Therefore, further validation is necessary, since low values of absorption coefficient of the lens for this wavelength should suggest much lower temperatures. Also, analysis has confirmed that the pupillary opening significantly affects the maximum temperature achieved in the eye, as is the case for the corneal beam diameter. On the other hand, an ambient temperature seems to have a negligible effect.

\section{References}

[1] Mainster, M.A., Ophthalmic applications of infrared lasers - thermal considerations. Investigative Ophthalmology \& Visual Science, 18(4), pp. 414-420, 1979.

[2] Amara, E., Numerical investigations on thermal effects of laser-ocular media interaction. International Journal of Heat and Mass Transfer, 38(13), pp. 2479-2488, 1995.

[3] Sbirlea, G. \& L'Huillier, J., A powerful finite element for analysis of argon 
laser iridectomy - influence of natural convection on the human eye. Transactions on BIomedicine and Health, 4, pp. 67-79, 1997.

[4] Chua, K.J., Ho, J.C., Chou, S.K. \& Islam, M.R., On the study of the temperature distribution within a human eye subjected to a laser source. International Communications in Heat and Mass Transfer, 32, pp. 1057-1065, 2005.

[5] Peratta, A., Electromagnetic modelling of human eye exposed to Conductive Keratoplasty. WIT Transactions in Biomedicine and Health, 12(VII), pp. 223-229, 2007.

[6] Cvetković, M., Poljak, D. \& Peratta, A., Thermal modelling of the human eye exposed to laser radiation. 2008 International Conference on Software, Telecommunications and Computer Networks, 10, pp. 16-20, 2008.

[7] Thompson, K., Ren, Q. \& Parel, J., Therapeutic and diagnostic application of lasers in ophthalmology. Proceedings of the IEEE, 80(6), 1992.

[8] Podol'tsev, A. \& Zheltov, G., Photodestructive effect of IR laser radiation on the cornea. Optics and Spectroscopy, 102(1), pp. 142-146, 2007.

[9] DeMarco, S.C., Lazzi, G., Liu, W., Weiland, J.D. \& Humayun, M.S., Computed SAR and thermal elevation in a $0.25-\mathrm{mm} 2-\mathrm{D}$ model of the human eye and head in response to an implanted retinal stimulator - Part I: Models and methods. IEEE Transactions on Antennas and Propagation, 51(9), pp. 2274 2285, 2003.

[10] Makous, W. \& Gould, J., Effects of lasers on the human eye. IBM Journal of Research and Development, 12(3), pp. 257-271, 1968.

[11] Krauss, J., Puliafito, C. \& Steinert, R., Laser interactions with the cornea. Survey of Ophthalmology, 31(1), pp. 37-51, 1986.

[12] Carroll, L. \& Humphreys, T.R., Laser-tissue interactions. Clinics in Dermatology, 24, pp. 2-7, 2006.

[13] Cvetković, M., Analysis of the Temperature Distribution to the Human Eye Exposed to Laser Radiation, 2009. Wessex Institute of Technology, Master Thesis.

[14] Pennes, H.H., Analysis of tissue and arterial blood temperatures in the resting human forearm. 1948. Journal of Applied Physiology, 85(1), pp. 5-34, 1998.

[15] Minkowycz, W.J., Sparrow, E.M. \& Murthy, J.Y., Handbook of Numerical Heat Transfer, Second Edition. John Willey \& Sons, Inc., New York, p. 1024, 2006.

[16] Scott, J., A finite element model of heat transport in the human eye. Physics in Medicine and Biology, 33(2), pp. 227-241, 1988.

[17] $\mathrm{Ng}$, E. \& Ooi, E., FEM simulation of the eye structure with bioheat analysis. Computer Methods and Programs in Biomedicine, 82, pp. 268-276, 2006.

[18] Ooi, E., Ang, W. \& Ng, E., Bioheat transfer in the human eye: A boundary element approach. Engineering Analysis with Boundary Elements, 31(6), pp. 494-500, 2007.

[19] Ng, E. \& Ooi, E., Ocular surface temperature: A 3D FEM prediction using bioheat equation. Computers in Biology and Medicine, 37, pp. 829-835, 2007. 